\title{
Improved Car-Following Model Considering Modified Backward Optimal Velocity and Velocity Difference with Backward-Looking Effect
}

\author{
Md. Anowar Hossain ${ }^{1}$, K. M. Ariful Kabir ${ }^{1,2}$, Jun Tanimoto ${ }^{1,3}$ \\ ${ }^{1}$ Interdisciplinary Graduate School of Engineering Sciences, Kyushu University, Kasuga-koen, Kasuga-shi, Fukuoka, Japan \\ ${ }^{2}$ Department of Mathematics, Bangladesh University of Engineering and Technology, Dhaka, Bangladesh \\ ${ }^{3}$ Faculty of Engineering Sciences, Kyushu University, Kasuga-koen, Kasuga-shi, Fukuoka, Japan \\ Email: *anowar.math.buet@gmail.com
}

How to cite this paper: Hossain, Md.A., Kabir, K.M.A. and Tanimoto, J. (2021) Improved Car-Following Model Considering Modified Backward Optimal Velocity and Velocity Difference with Backward-Looking Effect. Journal of Applied Mathematics and Physics, 9, 242-259.

https://doi.org/10.4236/jamp.2021.92018

Received: January 11, 2021

Accepted: February 17, 2021

Published: February 20, 2021

Copyright $\odot 2021$ by author(s) and Scientific Research Publishing Inc. This work is licensed under the Creative Commons Attribution International License (CC BY 4.0).

http://creativecommons.org/licenses/by/4.0/ (c) (i) Open Access

\begin{abstract}
In this paper, a new traffic flow model called the forward-backward velocity difference (FBVD) model based on the full velocity difference model is proposed to investigate the backward-looking effect by applying a modified backward optimal velocity using generalized backward maximum speed. The FBVD model belongs to the family of microscopic models that consider spatiotemporally continuous formulations. Neutral stability conditions of the discrete car-following model are derived using the linear stability theory. The stability analysis results prove that the modified backward optimal velocity has a significant positive effect in stabilizing the traffic flow. Through nonlinear analysis, a kink-antikink solution is derived from the modified Korteweg-de Vries equation of the FBVD model to explain traffic congestion of the model. The validity of this theoretical model is checked using numerical results, according to which traffic jams were found to have been significantly diminished by the introduction of the modified backward optimal velocity.
\end{abstract}

\section{Keywords}

Realistic Backward Optimal Velocity, Standerized Backward Maximum Speed, Honked Effect, Backward-Looking Effect, Car-Following Modelling

\section{Introduction}

In this day and age, one of the most challenging issues being faced worldwide is the mitigation of traffic congestion along with the development of urban life, 
especially in large cities. A number of researchers have been working toward finding a solution, and various traffic flow models have been developed. In general, traffic flow models can be classified into two types [1]: 1) the macroscopic model or continuum model, in which traffic flow is regarded as a stream of a compressible fluid and lows of conservation on mass that means what-is-called continuity law, and velocity or flux are established [2] [3] [4] [5] [6], and 2) the microscopic model or discrete model, in which every vehicle is treated as a self-driven particle composing a stream, and the main focus is to formulate the interactions between a vehicle and a vehicle, and a vehicle to road infrastructures [7] [8] [9] [10] [11]. As a general trend in the field, the microscopic model has been given more attention than the macroscopic model. The most investigated microscopic model is the car-following model, which presumes single-lane traffic flow [12]-[18]. A set of models as variants have been proposed to improve the car-following model, including the optimum velocity model (OVM), generalized force model (GFM), full velocity difference model (FVDM), multiple velocity difference model (MVDM), gas kinetic model, hydrodynamic lattice model, and cellular automated (CA) model [19]-[28].

Most of the car-following traffic flow models only concentrate on the forward direction of the focal vehicle. However, they do not completely and realistically represent traffic scenarios. If we simply consider the forward optimal velocity, the driver is considered to focus only on the preceding car and is never influenced by the following car. For example, Bando et al. [29] and Jiang et al. [30] [31] developed OVM and FDVM considering this presumption. In such types of model, the traffic system is fully controlled by the leading vehicles. Although, the traffic flow system is leaded by the leading car in real life traffic flow system, the following car can urge its focal for some space using the horn effect when following driver has not enough space to go forward. This realistic scenario has been absent in those models. In recent years, some studies have been undertaken in which the backward optimal velocity is considered along with the forward optimal velocity. Accordingly, the driver is considered to continuously monitor the following car as well as the preceding car, since the driver of the following car may honk the horn to encourage them to drive faster if they are frustrated by the velocity of the focal vehicle. Considering the backward-looking effect, Ge et al. [32] proposed a lattice hydrodynamic traffic flow model, and this kind of traffic flow model has since been universally studied [33] [34] [35] [36] [37]. At present, a number of models are being studied to account for the backward-looking effect based on various foundational models, including the lattice hydrodynamic model, continuum model, and car-following model. In these models, the backward-looking effect is introduced by a negative sign with the forward optimal velocity function except an excellent model proposed by Kuang et al. [38]. From the mathematical point of view, we think something different from this idea because velocity never be negative whether it is forward optimal velocity or backward optimal velocity. The main objective of our study is to introduce a honked effect by a non-negative backward optimal velocity function for generalized 
backward maximum speed which much more realistic than others proposed model.

In this study, we improved the car-following model to consider the backward-looking effect via the introduction of modified backward optimal velocity. To achieve this, we extended a conventional car-following model by introducing the concept of optimal velocity for the backward-looking effect in the form of the model. Herein, the backward optimal velocity is not defined as directly opposite (negative value) to the forward optimal velocity, but the backward and forward optimal velocities are instead considered to collaterally change in an inversely proportional manner with respect to the headway and backward gaps.

The rest of the paper is organized as follows. Conventional traffic flow models are introduced in Section 2. The proposed model is described in Section 3. A linear stability analysis of the present model is discussed in Section 4, and a nonlinear investigation is presented in Section 5. Numerical results of the model are provided in Section 6. Finally, Section 7 presents the conclusions.

\section{Background of Car-Following Models}

Traffic flow analysis is a special branch of mathematics and transportation engineering, which has been accelerated by the contribution of M. Bando. In 1995, Bando et al. [29] proposed a new model called OVM, the governing equation for which is as follows:

$$
\frac{\mathrm{d} v_{n}(t)}{\mathrm{d} t}=a\left[V\left(\Delta x_{n}(t)\right)-v_{n}(t)\right]
$$

where a represents the sensitivity of the driver, $v_{n}(t)$ denotes the velocity of car $n$ at time $t$, and $V\left(\Delta x_{n}(t)\right)$ is the optimal velocity function of the $n$th car, which depends on the headway distance between the $n$th car and the $n+1$ th car, represented by $\Delta x_{n}(t)=x_{n+1}(t)-x_{n}(t)$.

In OVM, unrealistic representations of acceleration and deceleration have been discovered. Helbing and Tilch [39] thus developed a traffic flow model called GFM to overcome the limitations of OVM. GFM is as follows:

$$
\frac{\mathrm{d} v_{n}(t)}{\mathrm{d} t}=a\left[V\left(\Delta x_{n}(t)\right)-v_{n}(t)\right]+\lambda \Delta v_{n}(t) H\left(-\Delta v_{n}\right)
$$

where $H$ is the Heaviside function, $\lambda$ is the sensitivity coefficient (different from a), and $\Delta v_{n}(t)=v_{n+1}(t)-v_{n}(t)$ is the velocity difference between $n+1$ th and $n$th car.

In 2001, Jiang et al. [30] [31] developed FVDM, which removed the negative velocity difference of the car that was introduced into GFM. The governing equation of FVDM is as follows:

$$
\frac{\mathrm{d} v_{n}(t)}{\mathrm{d} t}=a\left[V\left(\Delta x_{n}(t)\right)-v_{n}(t)\right]+\lambda \Delta v_{n}(t),
$$

where all the notations have the same meaning as in the original model. This FVDM is regarded as a standard model that is capable of assisting the investi- 
gation of a traffic flow field. Our main aim was to develop an extended car-following model based on FVDM by introducing the backward-looking effect in a modified form.

\section{Proposed Model}

Based on the FVDM [30], we propose an extended car-following model called the forward-backward velocity difference (FBVD) model, in which the backward optimal velocity is introduced in a modified form as follows:

$$
\frac{\mathrm{d} v_{n}(t)}{\mathrm{d} t}=a\left[p \cdot V_{F}\left(\Delta x_{n}(t)\right)+(1-p) V_{B}\left(\Delta x_{n-1}(t)\right)-v_{n}(t)\right]+\lambda \cdot \Delta v_{n}(t) .
$$

Here, $p$ is the weighted parameter for the forward optimal velocity. In other words, $p \&(1-p)$ means the driver concentration value for forward \& backward optimal velocity respectively. In case of real-life driving, the value backward concentration of a driver must be less than the forward concentration. So, we presumed the range of $p$ from 0.5 to 1.0. In Equation (4), $V_{F}$ and $V_{B}$ are the forward and backward optimal velocities, respectively. In fact, there have been several works than Ref. [40] accounting the backward optimal velocity function besides the forward one [41] [42] [43]. All of those pioneer works, despite being admirable, did presume the backward optimal velocity as a negative function, which seems unrealistic. It is because that when decreasing a gap with a following vehicle the focal vehicle would feel be urged by that following vehicle. Thus, the backward optimal velocity (OV) function should be defined as a positive as the forward one does. Unlike those studies, one brilliant work by Kuang et al. [38] previously posed a seminal model being analogous to what we introducing as below, which did not give, however, a general form as ours as below.

To this end, in this work, the forward and backward optimal velocities are defined by the following functions:

$$
\begin{gathered}
V_{F}\left(\Delta x_{n}(t)\right)=\frac{v_{\max }^{F}}{2}\left[\tanh \left(\Delta x_{n}(t)-h_{c}\right)+\tanh \left(h_{c}\right)\right], \\
V_{B}\left(\Delta x_{n-1}(t)\right)=\frac{v_{\max }^{B}}{2}\left[\tanh \left(h_{c}-\Delta x_{n-1}(t)\right)+\tanh \left(h_{c}\right)\right],
\end{gathered}
$$

where $v_{\max }^{F}$ and $v_{\max }^{B}$ denote the forward and backward maximum velocities, respectively, and $h_{c}$ is the safety distance. In general, $v_{\max }^{F} \neq v_{\max }^{B}$ is presumed. However, for the sake of simplicity, herein we studied two cases: case 1 . $v_{\max }^{F}=v_{\max }^{B}=2$ and case 2. $v_{\max }^{F}=2, v_{\max }^{B}=1$ whereas that safety distance $h_{c}=4$.

Figure 1 shows the characteristic behaviors of the forward and backward optimal velocities in the proposed model. The backward optimal velocity can be seen to reach its apex value when the forward optimal velocity is at its bottom value. The line graph of the forward optimal velocity increases with the decrease of the backward optimal velocity, and the forward and backward optimal velocities interchange at the position of the maximal slope of their respective functions 


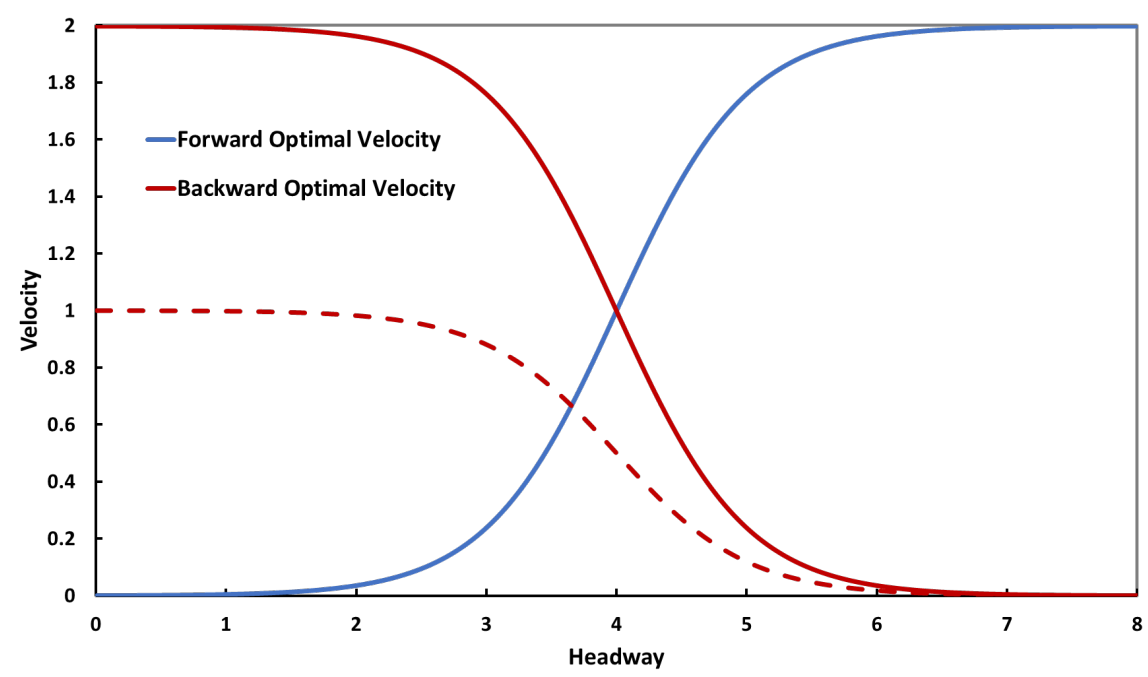

Figure 1. The optimal velocity profiles for forward and backward optimal velocities with respect to headway. The solid lines for persuming $v_{\max }^{F}=v_{\max }^{B}=2$ while dotted red line for $v_{\max }^{B}=1$.

due to the assumption we introduced above. Altogether, the forward and backward optimal velocities will evidently be equally balanced at any headway. In generally, forward and backward maximum velocities are integral part of forward and backward OV functions respectively and it has an important role. In this work we discussed forward and backward OV functions for both equal and unequal values of forward and backward maximum velocities. In Figure 1, the solid lines (red and blue) for equal value $\left(v_{\max }^{F}=v_{\max }^{B}=2\right)$ of forward and backward maximum velocities whereas that the red dotted and solid blue lines for unequal value $\left(v_{\max }^{F}=2, v_{\max }^{B}=1\right)$.

\section{Linear Stability Analysis}

A linear stability analysis was conducted to investigate the stability of the steady-state flow of the extended car-following model described in Equation (4) with Equations (5) and (6). The steady-state flow can be defined as a situation in which vehicles move with uniform headway $b$ with the forward and backward optimal velocities $\left(V_{F}(b)\right.$ and $V_{B}(b)$, respectively). Therefore, the steady-state solution of uniform flow is given by

$$
x_{n}^{0}(t)=b n+\left(p V_{F}(b)+(1-p) V_{B}(b)\right) t \text { and } b=L / N,
$$

where $L$ indicates the length of the road when presuming a cyclic boundary condition, and $N$ denotes the total number of cars.

Let us introduce a perturbed solution considering $y_{n}(t)$, defined as a small deviation from the steady-state solution $x_{n}^{0}(t)$ :

$$
x_{n}(t)=x_{n}^{0}+y_{n}(t) .
$$

Substituting Equations (7) and (8) into Equation (4), we then obtain the linearized formulation shown below: 


$$
\frac{\mathrm{d}^{2} y_{n}(t)}{\mathrm{d} t^{2}}=a\left[p V_{F}^{\prime}(b) \Delta y_{n}(t)+(1-p) V_{B}^{\prime}(b) \Delta y_{n-1}(t)-\frac{\mathrm{d} y_{n}(t)}{\mathrm{d} t}\right]+\lambda \frac{\mathrm{d} \Delta y_{n}(t)}{\mathrm{d} t}
$$

Here, $\Delta y_{n}(t)=y_{n+1}(t)-y_{n}(t), \quad V_{F}^{\prime}(b)=\left.\frac{\mathrm{d} V_{F}\left(\Delta x_{n}\right)}{\mathrm{d} \Delta x_{n}}\right|_{\Delta x_{n}=b}$, and $V_{B}^{\prime}(b)=\left.\frac{\mathrm{d} V_{B}\left(\Delta x_{n-1}\right)}{\mathrm{d} \Delta x_{n-1}}\right|_{\Delta x_{n-1}=b}$. Expanding $y_{n}(t)=\exp (i k n+z t)$, we obtain the following equation:

$$
z^{2}=a\left[p V_{F}^{\prime}(b)\left(\mathrm{e}^{i k}-1\right)+(1-p) V_{B}^{\prime}(b)\left(1-\mathrm{e}^{-i k}\right)-z\right]+\lambda z\left(\mathrm{e}^{i k}-1\right) .
$$

Suppose that $z=z_{1}(i k)+z_{2}(i k)^{2}+\cdots$. Substituting this value into Equation (10), the first- and second-order terms of $i k$ are as follows:

$$
\begin{gathered}
z_{1}=p V_{F}^{\prime}(b)+(1-p) V_{B}^{\prime}(b), \\
z_{2}=\frac{1}{a}\left[-z_{1}^{2}+\lambda z_{1}\right]-\left[-\frac{1}{2} p V_{F}^{\prime}(b)+(1-p) V_{B}^{\prime}(b)\right] .
\end{gathered}
$$

For a small disturbance, the traffic flow becomes unstable if the following condition is satisfied:

$$
a<\frac{2\left[p V_{F}^{\prime}(b)+(1-p) V_{B}^{\prime}(b)\right]^{2}-2 \lambda\left[p V_{F}^{\prime}(b)+(1-p) V_{B}^{\prime}(b)\right]}{p V_{F}^{\prime}(b)-(1-p) V_{B}^{\prime}(b)} .
$$

Hence, the neutral stability condition can be expressed as

$$
a=\frac{2\left[p V_{F}^{\prime}(b)+(1-p) V_{B}^{\prime}(b)\right]^{2}-2 \lambda\left[p V_{F}^{\prime}(b)+(1-p) V_{B}^{\prime}(b)\right]}{p V_{F}^{\prime}(b)-(1-p) V_{B}^{\prime}(b)} .
$$

This neutral stability condition [Equation (14)] is controlled by the parameters $p$ and $\lambda$. These parameters $p$ and $\lambda$ are determined by concentration of the driver and sensitivity (which determined by the driver's reaction capability for velocity difference) of the driver.

Figure 2 shows the results of the stability analysis presented above. Panel (a) illustrates a basic comparison of OVM, FVDM, and the FBVD model for $\lambda=0.1$. The backward-looking effect is influenced by the value of $v_{\max }^{B}$, as a result the linear stability curves of FBVD model varied for different value of $v_{\max }^{B}$. But these curves never go beyond the neutral stability curve of conventional OV and FVD models that means the OV and FVD models always resurved more unstable region than the proposed FBVD model, which reveals that our extended car-following model demonstrated better performance than the other two models with respect to mitigating the instability of traffic flow. Panel (b) shows stability plots of the FBVD model for $p=0.9,0.8,0.7$, and 0.6, presuming a fixed $\lambda=0.1$. For the fixed $\lambda=0.1$, the neutral stability curve gradually turns downwards as $p$ decreases, which represents a reduction of the unstable region due to the modified backward optimal velocity function. Panel (c) shows the neutral stability graphs for a fixed $\lambda=0.2$ with varying $p$. Here, the same tendency can observe for different $\lambda$. 
(a)

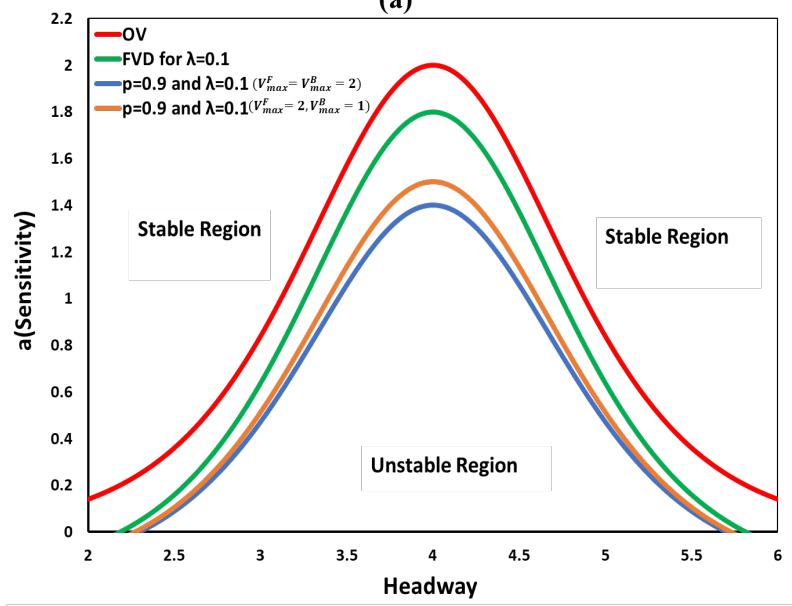

(b)

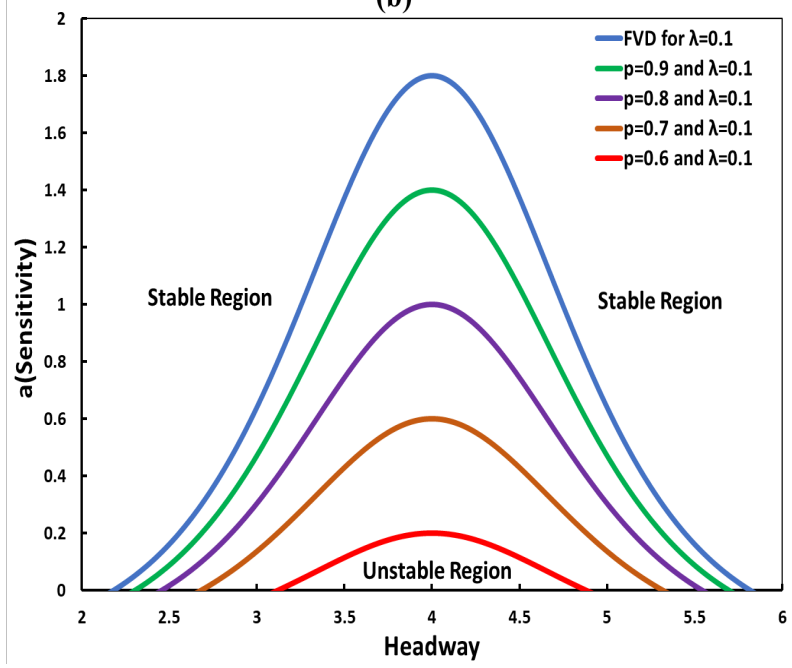

(c)

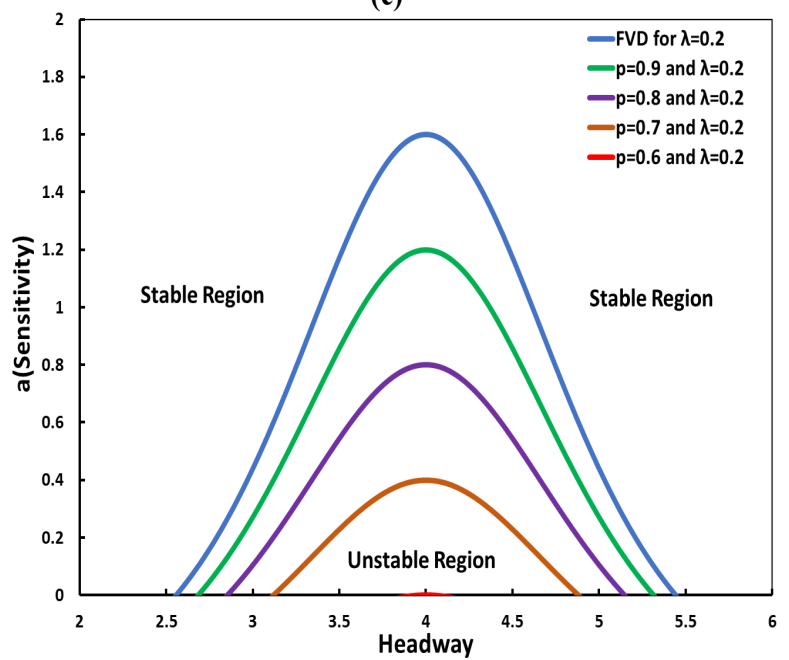

Figure 2. Comparison of the neutral stability diagrams among (a) OVM, FVDM, and the FBVD model for $\lambda=0.1$ with different $v_{\max }^{B}$; (b) FVDM with $\lambda=0.1$ and the FBVD model for $p=0.9,0.8,0.7$, and 0.6 with $\lambda=0.1$; and (c) FVDM with $\lambda=0.2$ and the FBVD model for $p=0.9,0.8,0.7$, and 0.6 with $\lambda=$ 0.2. In both panals (b) \& (c), $v_{\max }^{F}=v_{\max }^{B}=2$ are presumed. 
Since the backward-looking effect as well as the traffic flow stability may vary with $v_{\max }^{B}$, despite that, the backward-looking effect plays a significant role in mitigating the instability of traffic flow. Moreover, compared to the conventional FVDM under the same conditions, the FBVD model better realizes a stable flow for any trivial value of $v_{\max }^{B}$.

\section{Nonlinear Analysis}

In this section, a nonlinear analysis is performed, and a modified Korteweg-de Vries (mKdV) equation is derived to describe the kink-antikink wave solution.

First, let us introduce the headway form of Equation (4):

$$
\begin{aligned}
\frac{\mathrm{d}^{2} \Delta x_{n}(t)}{\mathrm{d} t^{2}}= & a\left[p\left(V_{F}\left(\Delta x_{n+1}(t)\right)-V_{F}\left(\Delta x_{n}(t)\right)\right)+(1-p)\left(V_{B}\left(\Delta x_{n}(t)\right)\right.\right. \\
& \left.\left.-V_{B}\left(\Delta x_{n-1}(t)\right)\right)-\frac{\mathrm{d} \Delta x_{n}(t)}{\mathrm{d} t}\right]+\lambda\left[\frac{\mathrm{d} \Delta x_{n+1}(t)}{\mathrm{d} t}-\frac{\mathrm{d} \Delta x_{n}(t)}{\mathrm{d} t}\right]
\end{aligned}
$$

Equation (15) leads us to the reductive perturbation method. Around the critical point $\left(a_{c}, h_{c}\right)$, a small positive scaling parameter $\varepsilon$ is introduced. Let us then define the slow scales $X$ and $T$ [44] [45], where the space variable $n$ and time variable $t$ are transformed as follows:

$$
X=\varepsilon(n+b t) \text { and } T=\varepsilon^{3} t \text { with } 0<\varepsilon \leq 1,
$$

where $b$ is a constant to be determined. The headway distance $\Delta x_{n}(t)$ can be defined as follows:

$$
\Delta x_{n}(t)=h_{c}+\varepsilon R(X, T) .
$$

We next substitute Equations (16) and (17) into Equation (15), and then, we expand the equation using a Taylor expansion of $\varepsilon$ up to the fifth order. We thus obtain the following nonlinear partial differential equation:

$$
\begin{aligned}
& \varepsilon^{2}\left(b-p V_{F}^{\prime}-(1-p) V_{B}^{\prime}\right) \partial_{X} R+\varepsilon^{3}\left[\frac{b^{2}}{a}-\frac{\lambda b}{a}-\frac{p}{2} V_{F}^{\prime}-\frac{1-p}{2} V_{B}^{\prime}\right] \partial_{X}^{2} R \\
& +\varepsilon^{4}\left[\partial_{T} R-\left\{\frac{1}{6}\left(p V_{F}^{\prime}+(1-p) V_{B}^{\prime}\right)+\frac{\lambda b}{2 a}\right\} \partial_{X}^{3} R-\frac{1}{2}\left(p V_{F}^{\prime \prime \prime}+(1-p) V_{B}^{\prime \prime \prime}\right) \partial_{X} R^{3}\right] \\
& +\varepsilon^{5}\left[\frac{2 b-\lambda}{a} \partial_{X} \partial_{T} R-\left\{\frac{1}{24}\left(p V_{F}^{\prime}+(1-p) V_{B}^{\prime}\right)+\frac{\lambda b}{6 a}\right\} \partial_{X}^{4} R\right. \\
& \left.-\frac{3}{4}\left(p V_{F}^{\prime \prime \prime}+(1-p) V_{B}^{\prime \prime \prime}\right) \partial_{X}^{2} R^{3}\right]=0
\end{aligned}
$$

where $V_{F}^{\prime}(b)=\left.\frac{\mathrm{d} V_{F}\left(\Delta x_{n}\right)}{\mathrm{d} \Delta x_{n}}\right|_{\Delta x_{n}=b}, \quad V_{F}^{\prime}(b)=\left.\frac{\mathrm{d} V_{F}\left(\Delta x_{n}\right)}{\mathrm{d} \Delta x_{n}}\right|_{\Delta x_{n}=b}$ and

$V_{F}^{\prime \prime \prime}(b)=\left.\frac{\mathrm{d}^{3} V_{F}\left(\Delta x_{n}\right)}{\mathrm{d} \Delta x_{n}^{3}}\right|_{\Delta x_{n}=b}, \quad V_{B}^{\prime \prime \prime}(b)=\left.\frac{\mathrm{d}^{3} V_{B}\left(\Delta x_{n-1}\right)}{\mathrm{d} \Delta x_{n-1}^{3}}\right|_{\Delta x_{n-1}=b}$.

Now, let us introduce $a_{c}=a\left(1+\varepsilon^{2}\right)$ as the neighbor to the critical point $\left(a_{c}, h_{c}\right)$ and consider $b=p V_{F}^{\prime}(b)+(1-p) V_{B}^{\prime}(b)$. The terms in Equation (18) 
containing second and third orders of $\varepsilon$ should be neglected; this allows us to simplify the equation as follows:

$$
\varepsilon^{4}\left(\partial_{T} R-g_{1} \partial_{X}^{3} R+g_{2} \partial_{X} R^{3}\right)+\varepsilon^{5}\left(g_{3} \partial_{X}^{2} R+g_{4} \partial_{X}^{4} R+g_{5} \partial_{X}^{2} R^{3}\right)=0,
$$

where the values of $g_{i}$ are given in Table 1 .

To derive the regularized equation, the following transformations are applied to Equation (19):

$$
T=\frac{1}{g_{1}} T^{\prime} \text { and } R=\sqrt{\frac{g_{1}}{g_{2}}} R^{\prime}
$$

The standard $\mathrm{mKdV}$ equation with a correction term $O(\varepsilon)$ is given as follows:

$$
\partial_{T^{\prime}} R^{\prime}-\partial_{X}^{3} R^{\prime}+\partial_{X} R^{\prime 3}+\varepsilon M\left[R^{\prime}\right]=0,
$$

where $M\left[R^{\prime}\right]=\frac{1}{g_{1}}\left[g_{3} \partial_{X}^{2} R^{\prime}+g_{4} \partial_{X}^{4} R^{\prime}+\frac{g_{1} g_{5}}{g_{2}} \partial_{X}^{2} R^{\prime 3}\right]$.

By eliminating the term $O(\varepsilon)$ from Equation (21), we obtain the kink-antikink solution of the $\mathrm{mKdV}$ equation:

$$
R_{0}^{\prime}\left(X, T^{\prime}\right)=\sqrt{c} \tanh \left[\sqrt{\frac{c}{2}}\left(X-c T^{\prime}\right)\right] .
$$

To determine the selected value of the propagation velocity $c$ for the kink solution in Equation (22), satisfying the following solvability condition is necessary [44]:

$$
\left(R_{0}^{\prime}, M\left[R^{\prime}\right]\right)=\int_{-\infty}^{\infty} \mathrm{d} X R_{0}^{\prime} M\left[R_{0}^{\prime}\right]=0,
$$

where $M\left[R_{0}^{\prime}\right]=M\left[R^{\prime}\right]$. To obtain the selected velocity $\mathcal{c}$, we perform the integration of Equation (23):

$$
c=\frac{5 g_{2} g_{3}}{2 g_{2} g_{4}-3 g_{1} g_{5}} .
$$

Hence, the kink-antikink solution of the $\mathrm{mKdV}$ equation can be derived as follows:

$$
R(X, T)=\sqrt{\frac{g_{1} c}{g_{2}}} \tanh \sqrt{\frac{c}{2}}\left(X-c g_{1} T\right) .
$$

Thus, the kink-antikink soliton solution of the headway for the original Equation (15) is

Table 1. The coefficients $g_{i}$ of the FBVD model.

$$
\begin{array}{cr}
g_{1}=\frac{1}{6}\left(p V_{F}^{\prime}+(1-p) V_{B}^{\prime}\right)+\frac{\lambda b}{2 a} \quad g_{2}=-\frac{1}{2}\left(p V_{F}^{\prime \prime \prime}+(1-p) V_{B}^{\prime \prime \prime}\right) & g_{3}=0 \\
g_{4}=\frac{1}{24}\left(p V_{F}^{\prime}+(1-p) V_{B}^{\prime}\right)+\frac{\lambda b}{6 a} & g_{5}=-\frac{3}{4}\left(p V_{F}^{\prime \prime \prime}+(1-p) V_{B}^{\prime \prime \prime}\right)
\end{array}
$$




$$
\Delta x_{n}=h_{c}+\sqrt{\frac{g_{1} c}{g_{2}}\left(\frac{a_{c}}{a}-1\right)} \tanh \sqrt{\frac{c}{2}\left(\frac{a_{c}}{a}-1\right)}\left[n+\left(1-c g_{1}\left(\frac{a_{c}}{a}-1\right) t\right)\right] .
$$

The amplitude $A$ of the solution is given by

$$
A=\sqrt{\frac{g_{1} c}{g_{2}}\left(\frac{a_{c}}{a}-1\right)} .
$$

The kink-antikink wave solution represents a coexisting phase that includes both free flow and congestion. The headway space is described by $\Delta x_{n}=h_{c} \pm A$.

\section{Numerical Simulations}

To prove the realistic characteristics of the proposed extended car-following traffic flow model, this section provides a number of numerical results. The fourth-order Runge-Kutta method [46] is used to solve Equation (4) to compute the numerical results of the FBVD model.

We ran a series of numerical simulations under a periodic boundary condition (cyclic boundary condition) with the following initial disturbance:

$$
\begin{gathered}
\Delta x_{n}(0)=\Delta x_{n}(1)=\Delta x_{0}=4.0, \text { for } n \neq 50,51, \\
\Delta x_{n}(0)=4.0-0.5, \text { for } n=50, \\
\Delta x_{n}(0)=4.0+0.5, \text { for } n=51 .
\end{gathered}
$$

Here, we assumed that the total number of cars was $N=100$, the length of the road was $L=200$, the sensitivity was $a=1$, and $b=L / N$.

Figure 3 shows a comparative analysis of the velocity fluctuations of all cars at time $t=1000 \mathrm{~s}$ for different models given several parameters sets of $p$ and $\lambda$. The amplitudes of the velocity fluctuations for (i) OVM, (ii) FVDM with $\lambda=0.1$, and (iii) FVDM with $\lambda=0.2$ were extremely high. At the same time, in the headway profile diagrams (panels (iv)-(ix)) of FBVD model for $p=0.9$ and 0.85 , the amplitudes of the velocity variations were significantly relaxed compared to the previous cases. The latter part (panels (vii)-(ix)) of Figure 3 demonstrated the stop-and-go phenomena heavily shrink for $v_{\max }^{B}=2$ with compare to $v_{\max }^{B}=1$ (panels (iv)-(vi)). But, in both cases $\left(v_{\max }^{B}=2\right.$ and $v_{\max }^{B}=1$ ) amplitude of stop-and-go phenomena always less than the conventional OV and FVD model, that means FBVD model performed a more stable traffic flow than these traditional models. Comparing panels (iv) with (vi) (for $v_{\max }^{B}=1$ ) and (vii) with (ix) (for $v_{\max }^{B}=2$ ), note that a larger $p$ ensured oppressing velocity fluctuation very much. In summary, Figure 3 proves that the FBVD model realizes a smoother traffic flow field compared to the cases presuming the conventional model OVM or the accepted FVDM. This is due to the introduction of the modified backward optimal velocity effect for generalized backward maximum speed, which successfully emulates the likely situation in which the vehicle following the focal vehicle honks its horn to urge the focal vehicle onwards, and the focal vehicle adjusts its velocity to the optimal one by considering not only the preceding gap but also the following gap. 
(i) OVM

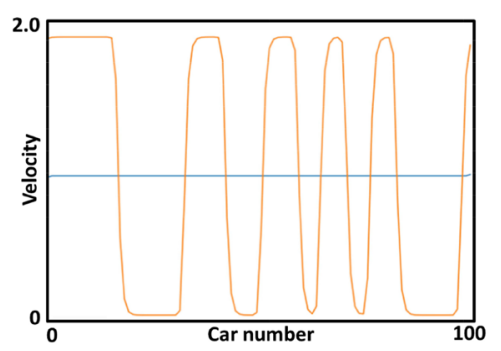

For $v_{\max }^{B}=1$

(iv) $\operatorname{FBVD}(p=0.9, \lambda=0.1)$

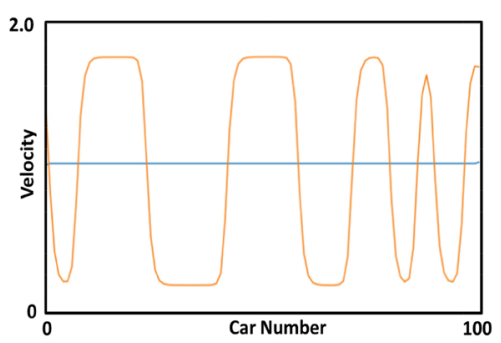

For $v_{\text {max }}^{B}=2$

(vii) $\operatorname{FBVD}(p=0.9, \lambda=0.1)$

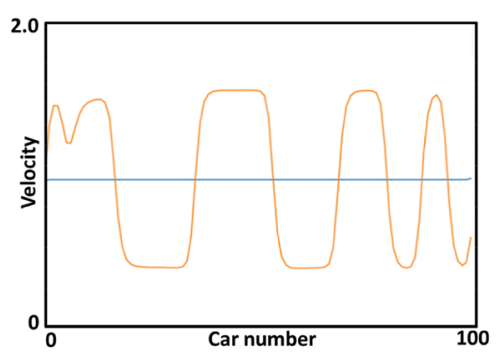

(ii) $\operatorname{FVDM}(\lambda=0.1)$



(v) $\operatorname{FBVD}(p=0.9, \lambda=0.2)$

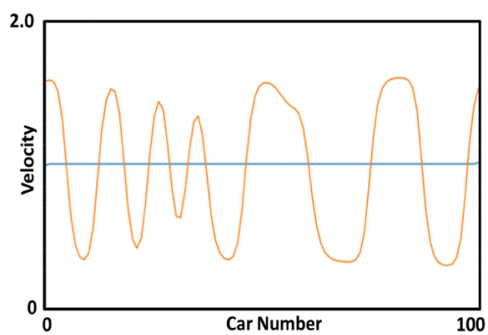

(viii) $\operatorname{FBVD}(p=0.9, \lambda=0.2)$

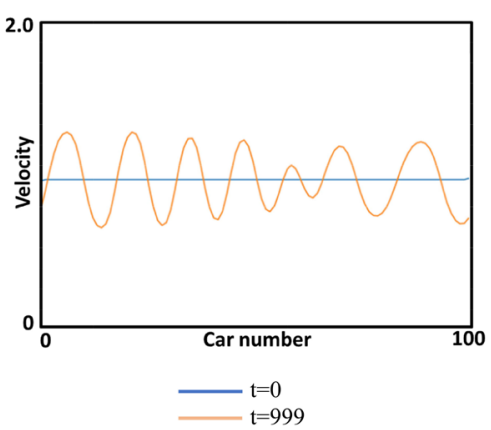

(iii) $\operatorname{FVDM}(\lambda=0.2)$

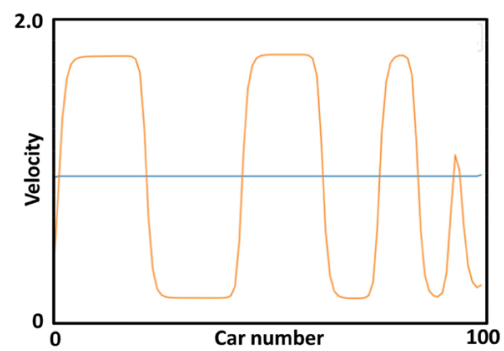

(vi) $\operatorname{FBVD}(p=0.85, \lambda=0.1)$

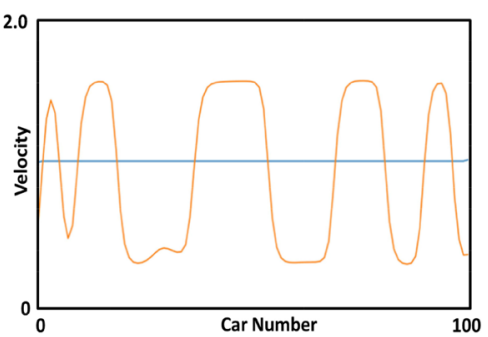

(ix) $\operatorname{FBVD}(p=0.85, \lambda=0.1)$

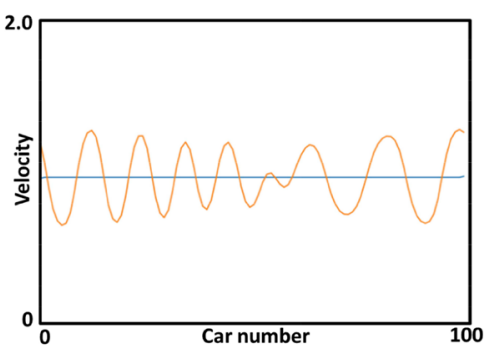

Figure 3. The velocity profile of all traffic at time $t=1000 \mathrm{~s}$ of (i) OVM, (ii) FVDM for $\lambda=0.1$, (iii) FVDM for $\lambda=0.2$, (iv)-(vi) FBVD model for $v_{\max }^{B}=1$ with $p=0.9 \& \lambda=0.1, p=0.9 \& \lambda=0.2$, and $p=0.85 \& \lambda=0.1$ rspectivly and (vii)-(ix) FBVD model for $v_{\max }^{B}=2$ with $p=0.9 \& \lambda=0.1, p=0.9 \& \lambda=0.2$, and $p=0.85 \& \lambda=0.1$ rspectivly.

To discuss the above numerical results from another viewpoint, the local velocity (shown in Figure 4) and local density (shown in Figure 5) are presented in a two-dimensional position-vs-time phase diagram for the same parameter settings (Figure 3). In the case of OVM and FVDM with $\lambda=0.1$ and 0.2 (panels (i)-(iii) in Figure 4 and Figure 5), spatially frequent stop-and-go waves, which can be detected as strap-like zones with quite a low velocity (Figure 4) and high density (Figure 5), were generated, and regions with relatively high velocity and low density emerged between the strap-like zones. Needless to say, such spatiotemporal concentrations of vehicles, which constitute a traffic jam, devastate the efficiency of traffic flow. Turning to our proposed model (panels (iv)-(ix)), although the combination of a higher $p$ and lower $\lambda$ was still present due to a 
traffic jam, we observed that the flow field was supported by moderate velocity and density over the entire domain. This was due to the introduction of our modified backward optimal velocity by considering the genaralized backward maximum speed into the backward-looking effect in the FBVD model, which ensured the dissolution of traffic jams and the realization of average velocity and density in spatiotemporal directions over the entire domain.

(i) OVM

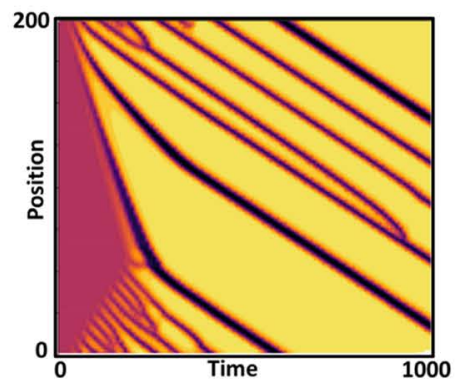

For $v_{\max }^{B}=1$

(iv) $\operatorname{FBVD}(p=0.9, \lambda=0.1)$

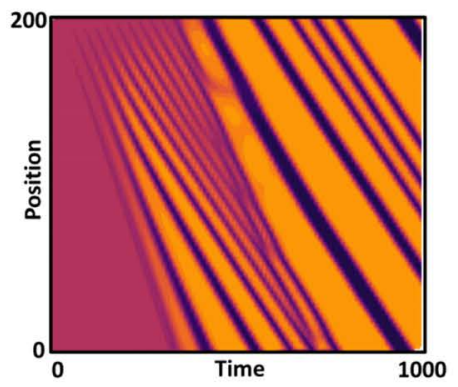

For $v_{\max }^{B}=2$

(vii) $\operatorname{FBVD}(p=0.9, \lambda=0.1)$

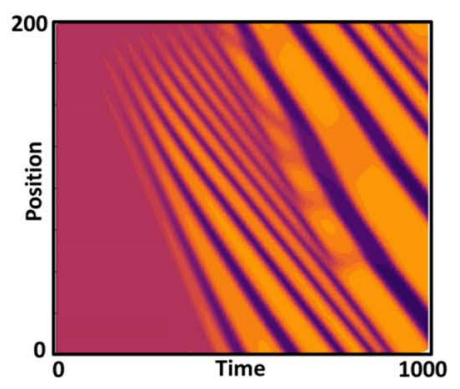

(ii) $\operatorname{FVDM}(\lambda=0.1)$

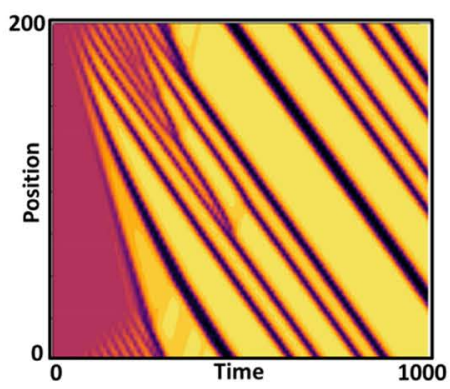

(v) $\operatorname{FBVD}(p=0.9, \lambda=0.2)$

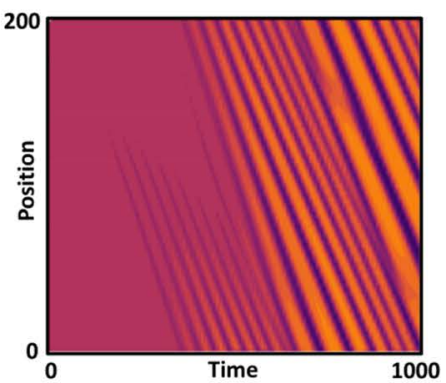

(viii) $\operatorname{FBVD}(p=0.9, \lambda=0.2)$

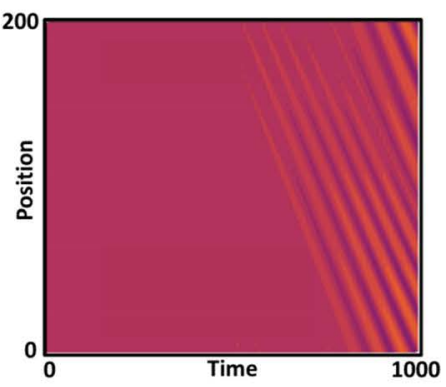

0.0



(iii) $\operatorname{FVDM}(\lambda=0.2)$

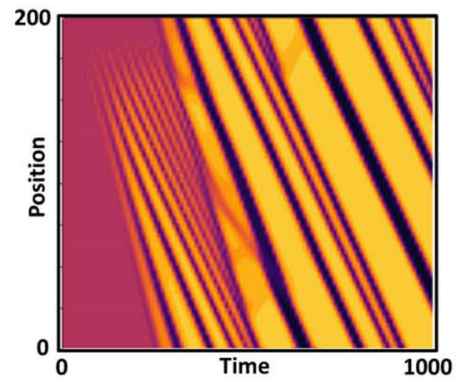

(vi) $\operatorname{FBVD}(p=0.85, \lambda=0.1)$

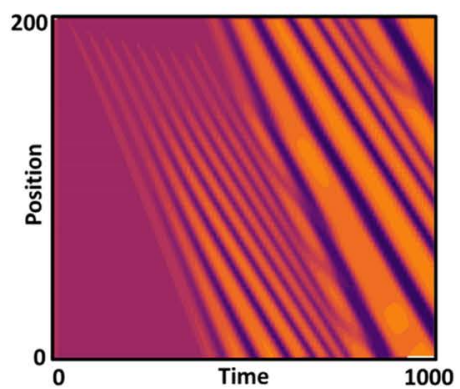

(ix) $\operatorname{FBVD}(p=0.85, \lambda=0.1)$

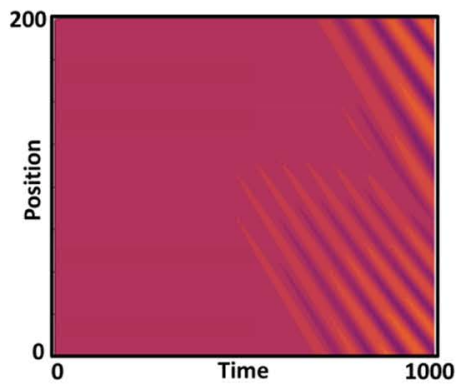

Figure 4. Car postion-vs-time diagram for the velocity of all cars of (i) OVM, (ii) FVDM for $\lambda=0.1$, (iii) FVDM for $\lambda=0.2$, (iv)-(vi) FBVD model for $v_{\max }^{B}=1$ with $p=0.9 \& \lambda=0.1, p=0.9 \& \lambda=0.2$, and $p=0.85 \& \lambda=0.1$ rspectivly and (vii)-(ix) FBVD model for $v_{\max }^{B}=2$ with $p=0.9 \& \lambda=0.1, p=0.9 \& \lambda=0.2$, and $p=0.85 \& \lambda=$ 0.1 rspectivly. 
(i) OVM

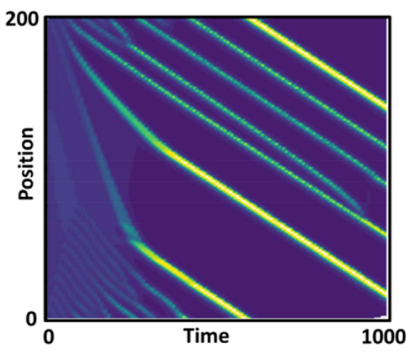

For $v_{\max }^{B}=1$

(iv) $\operatorname{FBVD}(p=0.9, \lambda=0.1)$

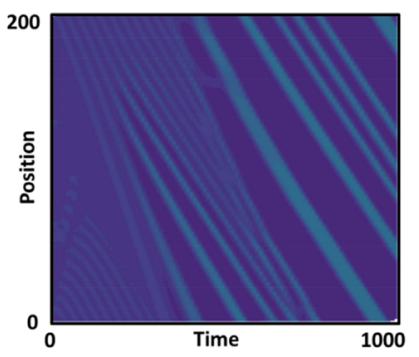

For $v_{\max }^{B}=2$

(vii) $\operatorname{FBVD}(p=0.9, \lambda=0.1)$

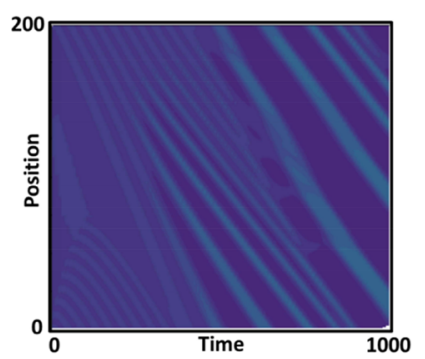

(ii) $\operatorname{FVDM}(\lambda=0.1)$

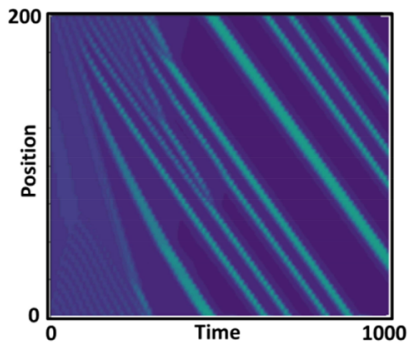

(v) $\operatorname{FBVD}(p=0.9, \lambda=0.2)$

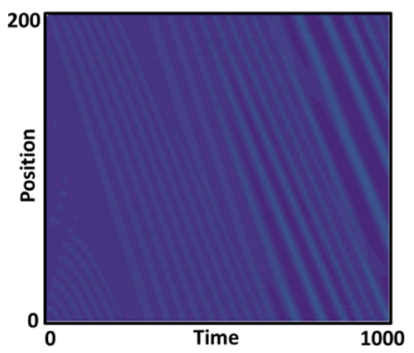

(viii) FBVD ( $p=0.9, \lambda=0.2)$

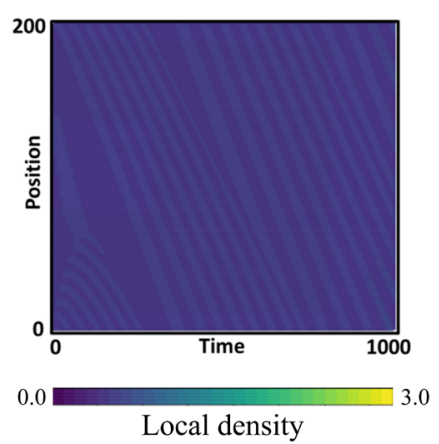

(iii) $\operatorname{FVDM}(\lambda=0.2)$

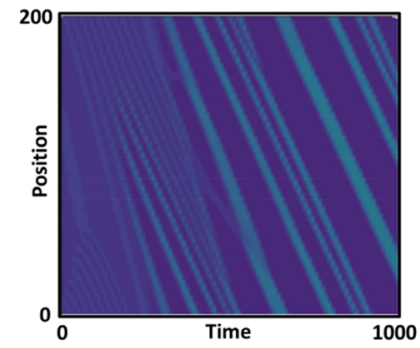

(vi) FBVD ( $p=0.85, \lambda=0.1)$

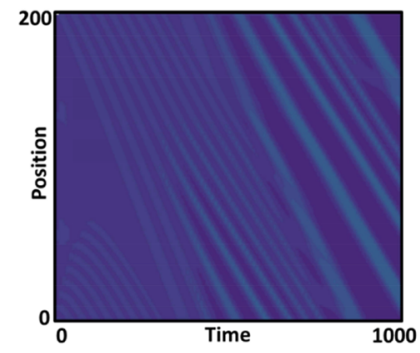

(ix) $\operatorname{FBVD}(p=0.85, \lambda=0.1)$



Figure 5. Car postion-vs-time diagram for the local density of all cars of (i) OVM, (ii) FVDM for $\lambda=$ 0.1 , (iii) FVDM for $\lambda=0.2$, (iv)-(vi) FBVD model for $v_{\max }^{B}=1$ with $p=0.9 \& \lambda=0.1, p=0.9 \& \lambda=$ 0.2 , and $p=0.85 \& \lambda=0.1$ rspectivly and (vii)-(ix) FBVD model for $v_{\max }^{B}=2$ with $p=0.9 \& \lambda=0.1$, $p=0.9 \& \lambda=0.2$, and $p=0.85 \& \lambda=0.1$ rspectivly.

Figure 6 displays the hysteresis loops of velocity and headway for the above discussed cases. The changes in color represent the passing time. Each of the conventional models (panels (i)-(iii)) attained a very large hysteresis loop, indicating a locally concentrated situation at an equilibrium flow state, which entails a poor efficiency in terms of traffic flow. In contrast, the FBVD model realized a much more relaxed situation in both cases for $v_{\max }^{B}=2$ and $v_{\max }^{B}=1$ showed in panels (iv)-(ix). To compare, panel (iv)-(vi) with (vii)-(ix), the FBVD model performed batter for $v_{\max }^{B}=2$ than $v_{\max }^{B}=1$. In particular, panels (v) and (vi) demonstrate that the FBVD model enabled a highly stable flow field from the initial state. 
(i) OVM

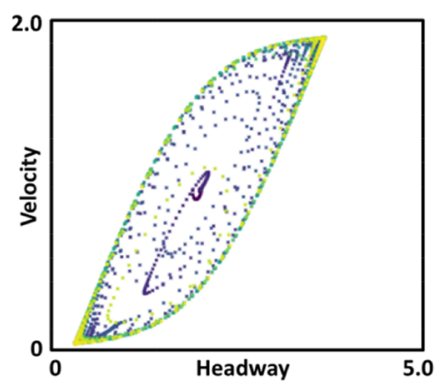

For $\boldsymbol{v}_{\max }^{B}=1$

(iv) $\operatorname{FBVD}(p=0.9, \lambda=0.1)$

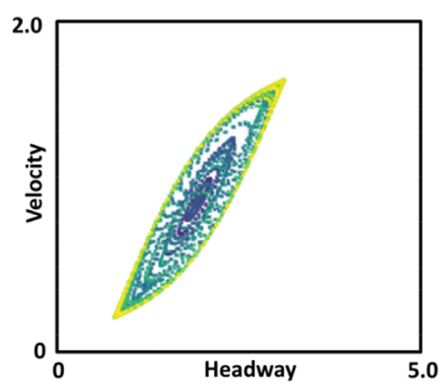

For $v_{\max }^{B}=2$

(iv) $\operatorname{FBVD}(p=0.9, \lambda=0.1)$

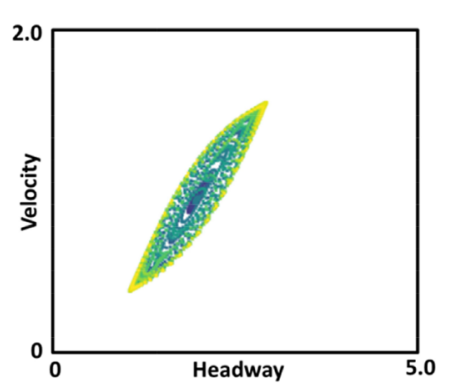

(ii) $\operatorname{FVDM}(\lambda=0.1)$

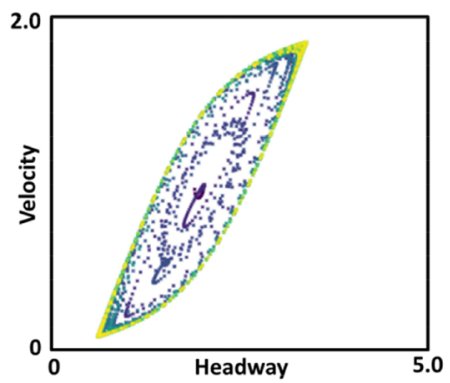

(v) $\operatorname{FBVD}(p=0.9, \lambda=0.2)$

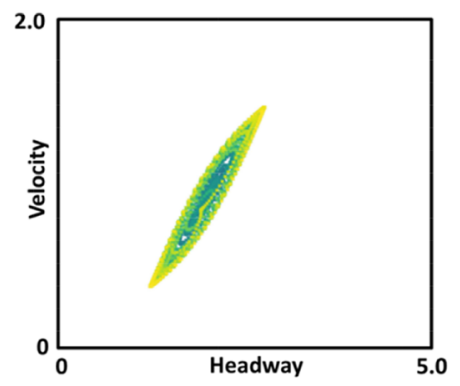

(v) $\operatorname{FBVD}(p=0.9, \lambda=0.2)$

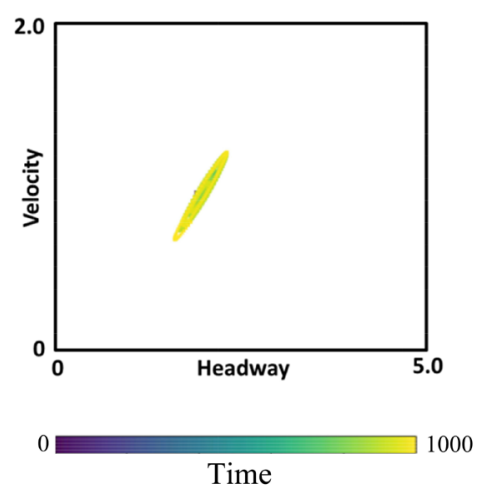

(iii) $\operatorname{FVDM}(\lambda=0.2)$

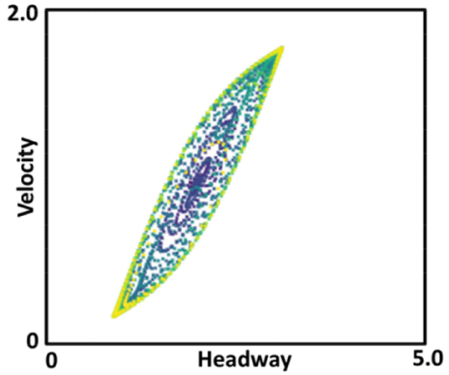

(vi) $\operatorname{FBVD}(p=0.85, \lambda=0.1)$

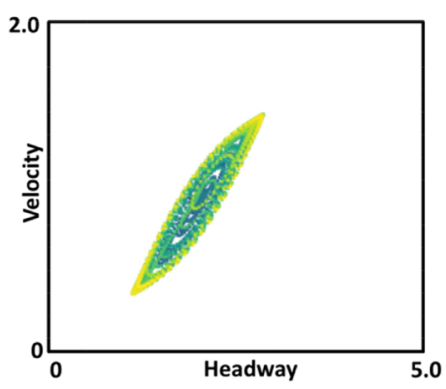

(vi) $\operatorname{FBVD}(p=0.85, \lambda=0.1)$

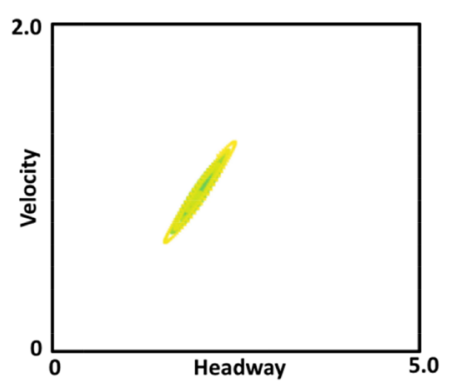

Figure 6. Hysteresis loop for the headway-vs-velocity of all cars of (i) OVM, (ii) FVDM for $\lambda=0.1$, (iii) FVDM for $\lambda=0.2$, (iv)-(vi) FBVD model for $v_{\max }^{B}=1$ with $p=0.9 \& \lambda=0.1, p=0.9 \& \lambda=0.2$, and $p=0.85 \& \lambda=0.1$ rspectivly and (vii)-(ix) FBVD model for $v_{\max }^{B}=2$ with $p=0.9 \& \lambda=0.1, p=0.9 \& \lambda=0.2$, and $p=0.85 \& \lambda=$ 0.1 rspectivly.

\section{Conclusions}

In this study, an improved car-following model called the FBVD model derived from the conventional OVM and FVDM is proposed to account for the backward-looking effect via the introduction of a modified backward optimal velocity function for generalized backward maximum speed. The FBVD model considers the likely situation in which the focal vehicle adjusts its velocity in response to not only the forward gap between it and the preceding vehicle but also 
the backward gap created by the following vehicle. This is justified by the reality that a driver tends to alter his velocity when being honked at by the following driver, who might be frustrated with the relatively low velocity of the focal vehicle.

Based on the formulation of the FBVD model, we explored a linear stability analysis and drew up a nonlinear analytic solution deriving the $\mathrm{mKdV}$ equation, and we also displayed numerical results generated by the model. All the results presented here indicate that the FBVD model affords a more stable flow field compared to OVM and FVDM. Thus, we can conclude that the backward-adjusting effect for velocity, as well as the forward one, plays a significant role in stabilizing the flow field.

Furthermore, please note that this finding may provide some hints in the design of automated vehicle control systems, which is a promising technology that will be developed over the next decade. In particular, this is because platoon running (a vehicle-cluster driving at relatively high speed) presumes simultaneous cooperative and mutual communication among both forward and backward vehicles.

\section{Acknowledgements}

This study was partially supported by Grant-in-Aid for Scientific Research from the Japan Society for the Promotion of Science (JSPS), Japan, KAKENHI (Grant Nos. JP 19KK0262 and JP 20H02314) awarded to Professor Tanimoto. We would like to express our gratitude to the organization.

\section{Conflicts of Interest}

The authors declare no conflicts of interest regarding the publication of this paper.

\section{References}

[1] Tanomoto, J. (2015) Traffic Flow Analysis Dovetailed with Evolutionary Game Theory. In: Tanimoto, J., Ed., Fundamentals of Evolutionary Game Theory and Its Applications, Vol. 6, Springer, Tokyo, 159-182. https://doi.org/10.1007/978-4-431-54962-8 5

[2] Nagatani, T. (1998) Modified KdV Equation for Jamming Transition in the Continuum Models of Traffic. Physica A: Statistical Mechanics and its Applications, 261, 599-607. https://doi.org/10.1016/S0378-4371(98)00347-1

[3] Tang, C.F., Jiang, R., Wu, Q.S., Wiwatanapataphee, B. and Wu, Y.H. (2007) Mixed Traffic Flow in Anisotropic Continuum Model. Transportation Research Record, 1999, 13-22. https://doi.org/10.3141\%2F1999-02

[4] Tang, T.Q., Huang, H.J. and Shang, H.Y. (2017) An Extended Macro Traffic Flow Model Accounting for the Driver's Bounded Rationality and Numerical Tests. Physica A: Statistical Mechanics and its Applications, 468, 322-333. https://doi.org/10.1016/j.physa.2016.10.092

[5] Tang, T.Q., Caccetta, L., Wu, Y.H., Huang, H.J. and Yang, X.B. (2014) A Macro 
Model for Traffic Flow on Road Networks with Varying Road Conditions. Journal of Advanced Transportation, 48, 304-317. https://doi.org/10.1002/atr.215

[6] Jiang, R., Wu, Q.S. and Zhu, Z.J. (2002) A New Continuum Model for Traffic Flow and Numerical Tests. Transportation Research Part B: Methodological, 36, 405-419. https://doi.org/10.1016/S0191-2615(01)00010-8

[7] Tian, J.F., Jiang, R., Jia, B., Gao, Z.Y. and Ma, S.F. (2016) Empirical Analysis and Simulation of the Concave Growth Pattern of Traffic Oscillations. Transportation Research Part B: Methodological, 93, 338-354. https://doi.org/10.1016/j.trb.2016.08.001

[8] Tian, J.F., Li, G.Y., Treiber, M., Jiang, R., Jia, N. and Ma, S. (2016) Cellular Automaton Model Simulating Spatiotemporal Patterns, Phase Transitions and Concave Growth Pattern of Oscillations in Traffic Flow. Transportation Research Part B: Methodological, 93, 560-575. https://doi.org/10.1016/j.trb.2016.08.008

[9] Tian, J.F., Jia, B., Ma, S., Zhu, C., Jiang, R. and Ding, Y. (2016) Cellular Automaton Model with Dynamical 2D Speed-Gap Relation. Transportation Science, 51, 807-822. https://doi.org/10.1287/trsc.2015.0667

[10] Tian, J.F., Jia, N., Zhu, N., Jia, B. and Yuan, Z.Z. (2014) Brake Light Cellular Automaton Model with Advanced Randomization for Traffic Breakdown. Transportation Research Part C: Emerging Technologies, 44, 282-298.

https://doi.org/10.1016/j.trc.2014.04.008

[11] Nagel, K. and Schreckenberg, M. (1996) Cellular Automaton Models of Traffic Flow along a Highway Containing a Junction. Journal of Physics A: Mathematical and General, 29, 3119-3127. https://doi.org/10.1088/0305-4470/29/12/018

[12] Newell, G.F. (1961) Nonlinear Effects in the Dynamics of Car Following. Operations Research, 9, 209-229. https://doi.org/10.1287/opre.9.2.209

[13] Li, X.P. and Ouyang, Y.F. (2010) Measurement and Estimation of Traffic Oscillation Properties. Transportation Research Part B: Methodological, 44, 1-14.

https://doi.org/10.1016/j.trb.2009.05.003

[14] Li, X.P., Cui, J.X., An, S. and Parsafard, M. (2014) Stop-and-GO Traffic Analysis: Theoretical Properties, Environmental Impacts and Oscillation Mitigation. Transportation Research Part B: Methodological, 70, 319-339.

https://doi.org/10.1016/j.trb.2014.09.014

[15] Tian, J.F., Treiber, M., Ma, S., Jia, B. and Zhang, W. (2015) Microscopic Driving Theory with Oscillatory Congested States: Model and Empirical Verification. Transportation Research Part B: Methodological, 71, 138-157. https://doi.org/10.1016/j.trb.2014.11.003

[16] Xie, D.F., Zhao, X.M. and He, Z.B. (2018) Heterogeneous Traffic Mixing Regular and Connected Vehicles: Modeling and Stabilization. IEEE Transactions on Intelligent Transportation Systems, 20, 2060-2071. https://doi.org/10.1109/TITS.2018.2857465

[17] Jiang, R., Jin, C.J., Zhang, H.M., Huang, Y.X., Tian, J.F., Wang, W., Hu, M.B., Wang, H. and Jia, B. (2018) Experimental and Empirical Investigations of Traffic Flow Instability. Transportation Research Part C: Emerging Technologies, 94, 83-98. https://doi.org/10.1016/j.trc.2017.08.024

[18] Reuschel, A. (1950) Fahrzeugbewegungen in der KolonneBeigleichformig beschleunigtem oder vertzogerten. Oesterreichisches Ingenieur-Archir, 4, 193-215.

[19] Tanimoto, J. and Nakamura, K. (2016) Social Dilemma Structure Hidden Behind Traffic Flow with Route Selection. Physica A: Statistical Mechanics and its Applica- 
tions, 459, 92-99. https://doi.org/10.1016/j.physa.2016.04.023

[20] Tanimoto, J. and An, X. (2019) Improvement of Traffic Flux with Introduction of a New Lane-Change Protocol Supported by Intelligent Traffic System. Chaos. Solitons \& Fractals, 122, 1-5. https://doi.org/10.1016/j.chaos.2019.03.007

[21] Hasebe, K., Nakayama, A. and Sugiyama, Y. (2004) Equivalence of Linear Response among Extended Optimal Velocity Models. Physical Review E, 69, Article ID: 017103. https://doi.org/10.1103/PhysRevE.69.017103

[22] Zhou, J., Shi, Z-K. and Cao, J-L. (2014) Nonlinear Analysis of the Optimal Velocity Difference Model with Reaction-Time Delay. Physica A: Statistical Mechanics and its Applications, 396, 77-87. https://doi.org/10.1016/i.physa.2013.11.007

[23] Ge, H.X., Meng, X.P., Ma, J. and Lo, S.M. (2012) An Improved Car-Following Model Considering Influence of Other Factors on Traffic Jam. Physics Letters A, 377, 9-12. https://doi.org/10.1016/j.physleta.2012.10.005

[24] Ge, H-X., Lv, F., Zheng, P-J. and Cheng, R-J. (2014) The Time-Dependent Ginzburg-Landau Equation for Car-Following Model Considering Anticipation-Driving Behavior. Nonlinear Dynamics, 76, 1497-1501. https://doi.org/10.1007/s11071-013-1223-y

[25] Li, Z., Li, W., Xu, S., Qian, Y. and Jian, S. (2015) Traffic Behavior of Mixed Traffic Flow with Two Kinds of Different Self-Stabilizing Control Vehicles. Physica A: Statistical Mechanics and its Applications, 436, 729-738.

https://doi.org/10.1016/j.physa.2015.05.090

[26] Jiang, R., Hu, M.B., Zhang, H.M., Gao, Z.Y., Jia, B. and Wu, Q.S. (2015) On Some Experimental Features of Car-Following Behavior and How to Model Them. Transportation Research Part B: Methodological, 80, 338-354. https://doi.org/10.1016/j.trb.2015.08.003

[27] Tang, T-Q., He, J., Yang, S-C. and Shang, H-Y. (2014) A Car-Following Model Accounting for the Driver's Attribution. Physica A: Statistical Mechanics and its Applications, 413, 583-591. https://doi.org/10.1016/j.physa.2014.07.035

[28] Hasebe, K. and Nakayama, A. and Sugiyama, Y. (2003) Dynamical Model of a Cooperative Driving System for Freeway Traffic. Physical Review E, 68, Article ID: 026102. https://doi.org/10.1103/PhysRevE.68.026102

[29] Bando, M., Haseba, K., Nakayama, A., Shibata, A. and Sugiyama, Y. (1995) Dynamical Model of Traffic Congestion and Numerical Simulation. Physical Review E, 51, 1035. https://doi.org/10.1103/PhysRevE.51.1035

[30] Jiang, R., Wu, Q.S. and Zhu, Z.J. (2001) Full Velocity Difference Model for a Car-Following Theory. Physical Review E, 64, Article ID: 017101. https://doi.org/10.1103/PhysRevE.64.017101

[31] Jiang, R. and Wu, Q.S. (2007) Two-Lane Totally Asymmetric Exclusion Processes with Particle Creation and Annihilation. Physica A: Statistical Mechanics and its Applications, 375, 247-256. https://doi.org/10.1016/j.physa.2006.08.025

[32] Ge, H.X. and Cheng, R.J. (2008) The "Backward Looking" Effect in the Lattice Hydrodynamic Model. Physica A: Statistical Mechanics and its Applications, 387, 6952-6958. https://doi.org/10.1016/j.physa.2008.05.060

[33] Jiang, C.T., Cheng, R.J. and Ge, H.X. (2018) An Improved Lattice Hydrodynamic Model Considering the "Backward Looking" Effect and the Traffic Interruption Probability. Nonlinear Dynamics, 97, 777-784. https://doi.org/10.1007/s11071-017-3908-0

[34] Hou, P., Yu, H., Yan, C. and Hong, J. (2017) An Extended Car-Following Model 
Based on Visual Angel and Backward Looking Effect. Chinese Journal of Physics, 55, 2092-2099. https://doi.org/10.1016/j.cjph.2017.08.027

[35] Tang, T.Q., Huang, H.J. and Shang, H.Y. (2015) Influences of the Driver's Bounded Rationality on Micro Driving Behavior, Fuel Consumption and Emissions. Transportation Research Part D: Transport and Environment, 41, 423-432. https://doi.org/10.1016/j.trd.2015.10.016

[36] Tang, T.Q., Zhang, J. and Liu, K. (2017) A Speed Guidance Model Accounting for the Driver's Bounded Rationality at a Signalized Intersection. Physica A: Statistical Mechanics and its Applications, 473, 45-52. https://doi.org/10.1016/j.physa.2017.01.025

[37] Tang, T.Q., Yi, Z.Y., Zhang, J. and Zheng, N. (2017) Modeling the Driving Behavior at a Signalized Intersection with the Information of Remaining Green Time. IET Intelligent Transport Systems, 11, 596-603. https://doi.org/10.1049/iet-its.2017.0191

[38] Kuang, H., Xu, Z.P., Li, X.L. and Lo, S.M. (2017) An Extended Car-Following Model Accounting for the Honk Effect and Numerical Tests. Nonlinear Dynamics, 87, 149-157. https://doi.org/10.1007/s11071-016-3032-6

[39] Helbing, D. and Tilch, B. (1998) Generalized Force Model of Traffic Dynamics. Physical Review E, 58, 133-138. https://doi.org/10.1103/PhysRevE.58.133

[40] Sun, D.H., Zhang, J.C. and Zhao, M. (2012) Effect of Looking Backward and Velocity Difference in an Extended Car Following Model. Journal of Sichuan University, 49, 115-120.

[41] Sun, D.H., Liao, X.Y. and Peng, G.H. (2011) Effect of Looking Backward on Traffic Flow in an Extended Multiple Car-Following Model. Physica A: Statistical Mechanics and its Applications, 390, 631-635. https://doi.org/10.1016/j.physa.2010.10.016

[42] Chen, C., Cheng, R. and Ge, H. (2019) An Extended Car-Following Model Considering Driver's Sensory Memory and the Backward Looking Effect. Physica A: Statistical Mechanics and its Applications, 525, 278-289.

https://doi.org/10.1016/j.physa.2019.03.099

[43] Ma, G., Ma, M., Liang, S., Wang, Y. and Zhang, Y. (2020) An Improved Car-Following Model Accounting for the Time-Delayed Velocity Difference and Backward-Looking Effect. Communications in Nonlinear Science and Numerical Simulation, 85, Article ID: 105221. https://doi.org/10.1016/j.cnsns.2020.105221

[44] Nagatani, T. (1998) Thermodynamic Theory for the Jamming Transition in Traffic Flow. Physical Review E, 58, 4271-4276.

https://doi.org/10.1103/PhysRevE.58.4271

[45] Nagatani, T. (2000) Density Waves in Traffic Flow. Physical Review E, 61, 3564-3570. https://doi.org/10.1103/PhysRevE.61.3564

[46] Hussain, K., Ismail, F. and Senu, N. (2016) Solving Directly Special Fourth-Order Ordinary Differential Equations Using Runge-Kutta Type Method. Journal of Computational and Applied Mathematics, 306, 179-199.

https://doi.org/10.1016/j.cam.2016.04.002 